\title{
Calcium Signaling in Neuronal Development
}

\author{
Sheila S. Rosenberg and Nicholas C. Spitzer \\ Neurobiology Section, Division of Biological Sciences, Kavli Institute for Brain and Mind, University of \\ California at San Diego, La Jolla, California 92093 \\ Correspondence: nspitzer@ucsd.edu
}

The development of the nervous system involves the generation of a stunningly diverse array of neuronal subtypes that enable complex information processing and behavioral outputs. Deciphering how the nervous system acquires and interprets information and orchestrates behaviors will be greatly enhanced by the identification of distinct neuronal circuits and by an understanding of how these circuits are formed, changed, and/or maintained over time. Addressing these challenging questions depends in part on the ability to accurately identify and characterize the unique neuronal subtypes that comprise individual circuits. Distinguishing characteristics of neuronal subgroups include but are not limited to neurotransmitter phenotype, dendritic morphology, the identity of synaptic partners, and the expression of constellations of subgroup-specific proteins, including ion channel subtypes.

$\mathrm{H}$ ow is the diversity of neuronal subtypes generated during development? Intrinsic genetic programs, extracellular signals, and both spontaneous and experience-dependent activity all contribute to the development of a functional nervous system. Here we focus on the role of activity, and specifically calciummediated signaling, in the generation of neuronal diversity. Brief elevations of intracellular calcium levels, also referred to as calcium transients, have been implicated in the regulation of various stages of neuronal development, including proliferation, migration, differentiation, and survival. Calcium-mediated regulation of neuronal proliferation and migration has been recently reviewed (Spitzer 2006; Platel et al. 2008). Here we address the role of calcium transients in neuronal differentiation. Calcium-mediated signaling contributes to the specification of neuronal subtype through the regulation of axonal pathfinding, dendritic growth and arborization, and specification of neurotransmitter subtype (Fig. 1).

How is it that a single cation can regulate so many different aspects of differentiation? In addition, how does calcium-mediated regulation of each distinguishing neuronal feature lead to specific phenotypic characteristics in different cell types? Although the answers to these questions are not yet fully resolved, innovative work from many groups has helped establish that the spatial and temporal characteristics of activity are important for conferring specificity in calcium-mediated signaling. Therefore we begin this review by describing characteristics of calcium transients in the embryonic nervous system. Next, we review evidence demonstrating that different patterns of calcium transients

Editors: Martin Bootman, Michael J. Berridge, James W. Putney, and H. Llewelyn Roderick

Additional Perspectives on Calcium Signaling available at www.cshperspectives.org

Copyright (C) 2011 Cold Spring Harbor Laboratory Press; all rights reserved; doi: 10.1101/cshperspect.a004259

Cite this article as Cold Spring Harb Perspect Biol 2011;3:a004259 


\section{S.S. Rosenberg and N.C. Spitzer}

Subcellular location of ion channels and receptors

(e.g., dendrites; cell body; axon)

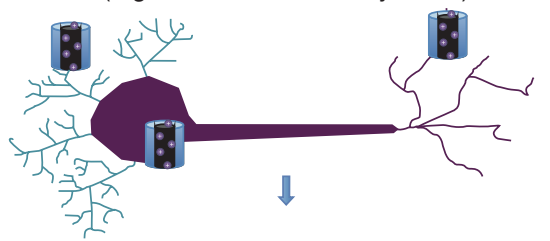

Identity of ion channels and receptors

(e.g., calcium-permeable channels; glutamate receptors; guidance receptors; neurotransmitter receptors)

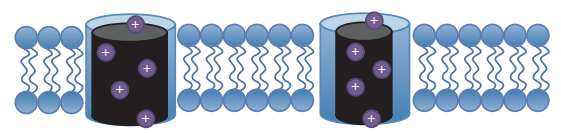

$\Downarrow$

Pattern of calcium transients (e.g., burst duration; interburst interval)

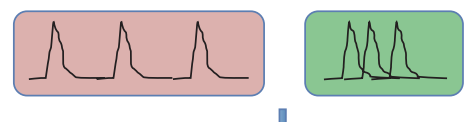

$\downarrow$

Identity, expression pattern, and activation state of transduction machinery

Calcium-dependent kinases (e.g., CaMKII)

Regulation of transcription

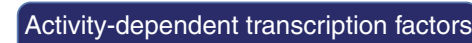
(e.g., cJun; Lmx1b)
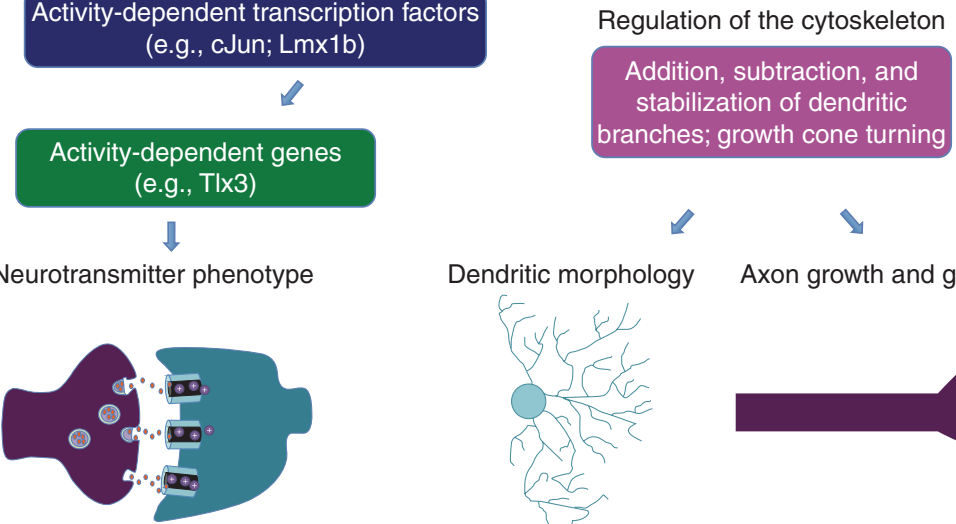
branches; growth cone turning

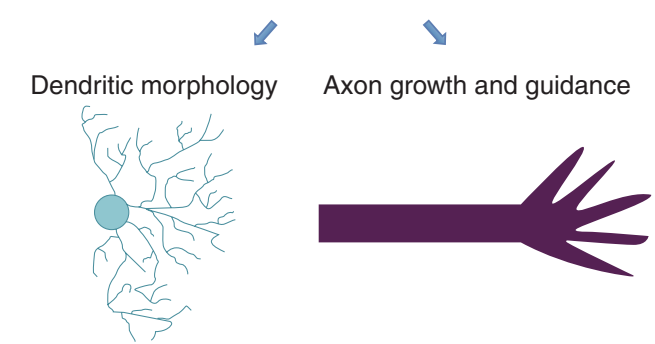

Figure 1. Providing specificity for calcium signaling in neuronal differentiation. Calcium transients direct neuronal differentiation by regulating neurotransmitter phenotype, dendritic morphology, and axonal growth and guidance. Factors dictating intracellular calcium dynamics include the subcellular location of ion channels within the neuron and the neuron-specific constellation of ion channels and receptors expressed by individual cells. The location and identity of these channels and receptors influence the timing and frequency of calcium transients and determine whether the changes in calcium concentration occur in a global or localized fashion. Spatiotemporal patterns of calcium transients select the downstream mechanisms involved in neuronal differentiation. Calcium transients activate enzymes that transduce ionic signals into biochemical ones. These enzymes impact differentiation either through transcriptional mechanisms or by the regulation of cytoskeletal dynamics. Activation or repression of transcription factors controls neurotransmitter expression whereas cytoskeletal remodeling regulates axon and dendrite morphogenesis. 
regulate distinct aspects of neuronal differentiation. Additionally, we discuss some downstream mechanisms involved in calcium-mediated effects on neuronal differentiation, including the activation of specific transcription factors and cytoskeletal rearrangements. We also review some of the identified calcium-binding partners and specific downstream targets that help to convey specificity in calcium signaling. The studies discussed here show that the temporal dynamics of calcium transients and the mode of calcium entry into the neuron influence the specific effects of calcium signaling in neuronal development. Based on these findings, it is likely that the mechanisms regulating calcium channel expression and localization play a fundamental role in regulating calcium-driven differentiation and the determination of neuronal subtype. Therefore, we conclude with findings that provide insight into calcium-dependent mechanisms regulating the subcellular targeting of calcium-permeable channels.

\section{SPATIOTEMPORAL CHARACTERISTICS OF CALCIUM TRANSIENTS DURING EARLY NEURONAL MATURATION}

Both spontaneous and experience-driven patterns of activity influence nervous system development. In experience-mediated activity, the number and frequency of calcium transients are responsible for conveying information about the stimulus intensity. Different patterns of calcium spike activity, and the resulting variations in ionic flux, lead to specific outcomes for neuronal development and plasticity. Similarly, the timing of expression of spontaneous activity is important for multiple aspects of neuronal development. Spontaneous activity, occurring in the absence of sensory input, takes place both before and after the formation of synaptic connections. The mechanisms involved in spontaneous synaptically mediated activity have been recently reviewed (Blankenship and Feller 2010).

We focus here on spontaneous activity present in the nervous system prior to the formation of synaptic connections. Remarkably, these early forms of activity have significant roles in directing neuronal differentiation. In an effort to understand the functional importance of early forms of spontaneous activity, calcium transients have been characterized in embryonic Xenopus spinal neurons beginning at the time of neural tube closure. These neurons show two distinct types of calcium transients: calcium spikes and calcium waves $(\mathrm{Gu}$ et al. 1994; Spitzer et al. 1994; Gu and Spitzer 1995, 1997). Calcium spikes involve a rapid increase in calcium concentration generated by calcium-dependent action potentials and calcium-induced calcium release, quickly followed by a stereotyped double exponential decay with time constants of $\sim 10 \mathrm{sec}$ and $\sim 3 \mathrm{~min}$. As measured by calcium imaging with Fura-2, calcium spikes raise intracellular calcium from $10^{-7}$ to $10^{-6} \mathrm{M}$, in $<5 \mathrm{sec}$ (Gu et al. 1994).

In contrast to the stereotyped pattern of calcium spikes, calcium waves show variability in duration, with rise and decay times lasting on the order of half a minute to several minutes in duration. Calcium waves also show a more moderate increase in fluorescence intensity when assessed with calcium imaging. In addition to these differences in amplitude and duration, spikes and waves also differ in their spatial localization within neurons. Consistent with their initiation by calcium-dependent action potentials, calcium spikes propagate rapidly throughout the cell. In contrast, calcium waves occur in both the soma and the growth cone, but frequently remain sequestered in the compartment in which they are generated. These two types of calcium transients also exert different effects on neuronal differentiation. Calcium spikes regulate neurotransmitter specification, whereas calcium waves regulate the rate of axon extension, indicating that the spatiotemporal features of spontaneous activity may provide functional specificity.

Spontaneous activity prior to the formation of synaptic networks has been observed in multiple areas of the nervous system and in multiple species. In the developing mammalian brain, spontaneous transients are present in slices of the visual, somatosensory, and frontal cortex at postnatal day 0 (Yuste et al. 1992; O’Donovan 1999). In the developing chick retina, calcium 
transients appear as early as embryonic day eight (E8) (Catsicas et al. 1998). These signals are also present in cultured chick dorsal root ganglion (DRG) neurons isolated at E8-E11 (Gomez et al. 1995). These neurons generate calcium transients in their growth cones that regulate the rate of growth cone migration, consistent with the idea that the subcellular location of activity patterns influences the effect of calcium signaling on neuronal differentiation. In the following sections, we discuss studies that provide additional insight into the spatiotemporal characteristics of calcium transients and the types of downstream mechanisms that confer specificity in the regulation of neuronal differentiation.

\section{CALCIUM-MEDIATED SPECIFICATION OF NEUROTRANSMITTER PHENOTYPE}

Early studies of cultured embryonic Xenopus spinal neurons showed a role for calcium spikes in the regulation of GABA immunoreactivity. Elimination of calcium spikes by the removal of extracellular calcium, blockade of calcium channels, or by the addition of the calcium chelator BAPTA, significantly reduced the number of GABAergic neurons (Holliday et al. 1991; Gu and Spitzer 1995). In the absence of extracellular calcium, artificial calcium spiking generated by pulses of $\mathrm{KCl}$ and $\mathrm{CaCl}_{2}$ was sufficient to rescue the GABAergic phenotype ( $\mathrm{Gu}$ and Spitzer 1995). These findings show that spontaneous calcium spikes are necessary and sufficient for the specification of neurotransmitter expression in developing Xenopus spinal neurons. What is the mechanism by which calcium signaling regulates GABA expression? In spinal neurons cultured at early stages of development, the release of calcium from intracellular stores is necessary for the generation of calcium spikes. Experimental depletion of intracellular calcium stores leads to a reduction in calcium spiking, and a decrease in the number of GABAergic cells (Holliday et al. 1991). These studies indicate that in addition to the influx of extracellular calcium, release of intracellular calcium stores is also necessary for neuronal differentiation. Together, these findings lead to a model in which the influx of extracellular calcium induces the release of calcium from intracellular stores, resulting in an increase in the number of GABAergic cells. Calcium-mediated regulation of GABA expression appears to occur via a frequency-dependent mechanism, as shown by removing extracellular calcium and stimulating cultured neurons with different frequencies of calcium spikes ( $\mathrm{Gu}$ and Spitzer 1995; Watt et al. 2000). Spike stimulation mimicking the frequency of spontaneous transients was shown to be most effective in replicating the effects of spontaneous transients on neurotransmitter expression.

The regulation of GABAergic phenotype by calcium spike frequency prompts a number of interesting questions. How does the frequency of calcium spikes modulate neurotransmitter specification? Is spike frequency responsible for regulating gene expression? Are diverse frequencies of calcium transients involved in generating the diversity of neuronal subtypes present in the nervous system? Do specific frequencies elicit the expression of specific neurotransmitters in different neuronal subtypes? Several of these questions were addressed by imaging the calcium transients of specific classes of neurons in the developing Xenopus spinal cord (Borodinsky et al. 2004). Four different neuronal subtypes-Rohon-Beard sensory neurons, ventral interneurons, ventral motor neurons, and dorsolateral interneurons-show unique patterns of spontaneous calcium spiking and disparate neurotransmitter phenotypes. For example, in the developmental period prior to synapse formation, RohonBeard neurons show a relatively low spike frequency and express glutamate, whereas motor neurons show a gradual increase in spike frequency and express acetylcholine. These results indicate a potential relationship between spiking activity and the expression of various neurotransmitters, which is consistent with activitydependent regulation of GABA expression. The role of spontaneous activity in regulating neurotransmitter phenotype was investigated using ion channel misexpression to manipulate activity and the resulting calcium transients (Borodinsky et al. 2004). Injection of voltage-gated 
sodium channel transcripts into Xenopus embryos at the two-cell stage results in a global increase in spiking activity and is accompanied by a decrease in the number of neurons expressing the excitatory transmitters glutamate and acetylcholine, as well as a corresponding increase in the number of neurons expressing the inhibitory transmitters glycine and GABA. In contrast, injection of inwardly rectifying potassium channel transcripts results in an overall decrease in the extent of calcium spiking, accompanied by a decrease in the numbers of neurons expressing inhibitory transmitters and an increase in the expression of excitatory transmitters by neurons other than sensory and motor neurons. These results indicate the existence of a homeostatic relationship between calcium signaling and neurotransmitter expression (Fig. 2A).

Changes in neurotransmitter specification that serve to readjust the relative degree of excitation and inhibition represent an intriguing strategy by which the nervous system can preserve a homeostatic balance in overall neuronal activity. Additionally, the activity-dependent acquisition of a novel neurotransmitter profile reveals a previously unexpected degree of plasticity in neuronal phenotype. This plasticity is also reflected in the matching of appropriate postsynaptic receptors to neurons expressing novel neurotransmitters (Borodinsky and Spitzer 2007). Studies of the developing Xenopus neuromuscular junction (NMJ) reveal that multiple neurotransmitter receptors are initially expressed in skeletal muscle. As development proceeds, muscles are innervated by neurons expressing acetylcholine, and receptors not responding to acetylcholine are normally down-regulated. Calcium-spike mediated expression of novel neurotransmitters results in the retention of alternate receptor subtypes at the NMJ. These findings support the likelihood of a functionally relevant role for neurons that undergo activity-induced acquisition of novel neurotransmitters.

Activity-dependent regulation of neurotransmitter phenotype raises new questions about the mechanisms involved in calciummediated neuronal specification. What are the triggers for spontaneous activity? Paracrine action of GABA and glutamate, secreted prior to synapse formation, drives spontaneous activity in the Xenopus neural tube (Root et al. 2008), and expression of the $\beta$-subunit of the sodium pump appears to be required for the generation of spontaneous calcium spikes (Chang and Spitzer 2009). What are the downstream mechanisms responsible for activity-dependent neurotransmitter respecification? Recent studies show that the activity-dependent regulation of GABAergic and glutamatergic expression in Xenopus tropicalis is mediated through the expression of the $t l \times 3$ homeobox gene (Marek et al. 2010). In mice, $t l x 3$ has been shown to act as a transcriptional selector gene, responsible for both the inhibition of GABAergic development and the up-regulation of the glutamatergic phenotype in the dorsal horn of the embryonic spinal cord (Cheng et al. 2004, 2005). In Xenopus, morpholino knockdown of tlx3 expression was sufficient to block the decrease in GABAergic neurons and corresponding increase in glutamatergic neurons that results from inhibition of activity. Additionally, overexpression of $t l x 3$ blocked the increase in GABA-expressing cells and decrease in glutamate-expressing cells that results from a global increase in activity. These results indicate that $t l \times 3$ expression mediates the activity-dependent regulation of glutamatergic and GABAergic cell fate. The use of luciferase transcription assays showed that the effect of calcium activity on tlx3 expression is mediated through the activity-dependent phosphorylation of the cJun transcription factor. An increase in activity leads to an increase in cJun phosphorylation and activation of cJun, which suppresses tlx3 expression by binding to a noncanonical cAMP responsive element (CRE) within the tl $x 3$ promoter. This results in an increase in the number of GABAergic cells and a decrease in the number of glutamatergic neurons. In contrast, a decrease in activity causes a decrease in the number of neurons showing phosphorylated cJun, and a decrease in the number of GABAergic cells and an increase in the number of glutamatergic cells (Fig. 2B). Together, these findings show that spontaneous activity and 


\section{S.S. Rosenberg and N.C. Spitzer}

A Activity-dependent changes in calcium signaling regulate neurotransmitter specification

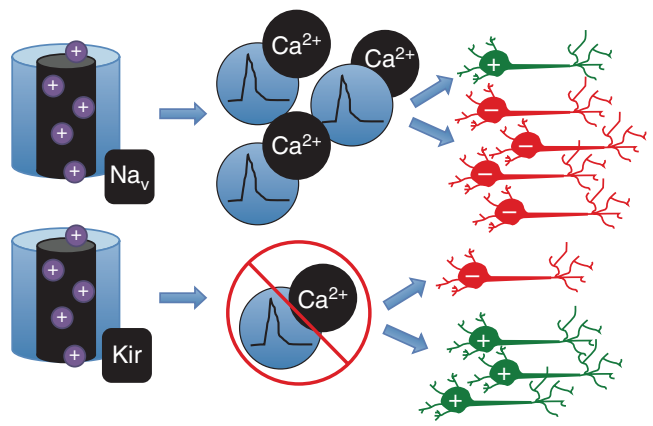

B Calcium signaling modulates transcriptional regulation of neurotransmitter specification
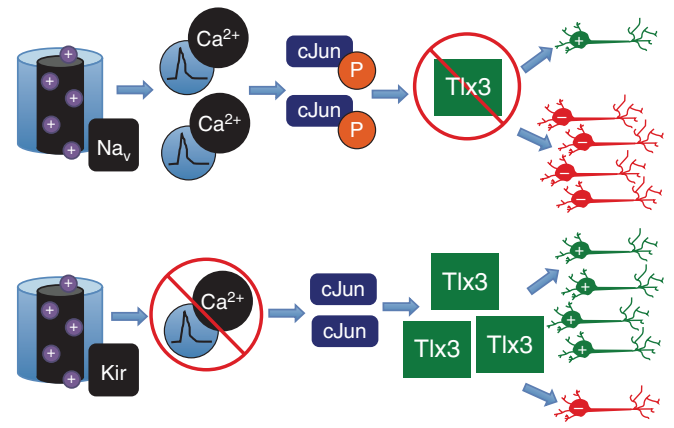

C Experience-dependent calcium signaling regulates neurotransmitter respecification
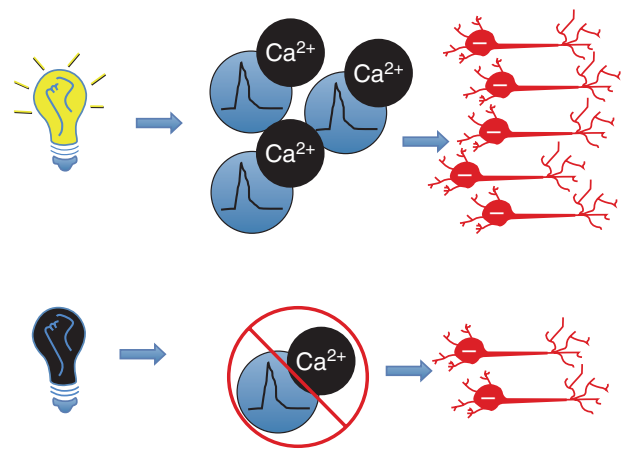

Figure 2. Activity-dependent neurotransmitter specification at early stages of neuronal development. (A) Prior to synapse formation in the embryonic spinal cord, overexpression of voltage-gated sodium channels $\left(\mathrm{Na}_{\mathrm{v}}\right)$ increases the incidence and frequency of calcium spikes that lead to a decrease in the number of neurons expressing excitatory transmitters (in green) and an increase in the number of neurons expressing inhibitory transmitters (in red). Overexpression of inward rectifier potassium channels (Kir) decreases calcium spiking and produces the opposite effect on transmitter specification. (B) Increasing the incidence and frequency of calcium spikes in the embryonic spinal cord leads to phosphorylation of the cJun transcription factor that binds to the promoter of the $t l x 3$ selector gene and suppresses its expression; this results in a decrease in the number of glutamatergic neurons (in green) and an increase in the number of GABAergic neurons (in red). Suppressing spike production leads to dephosphorylation of cJun that no longer represses $t l x 3$ expression; this results in an increase in the number of glutamatergic neurons and a decrease in the number of GABAergic neurons. $(C)$ After synapse formation in the postembryonic brain, bright light illumination increases calcium spike activity that leads to an increase in the number of hypothalamic neurons that express dopamine (in red), which acts as an inhibitory transmitter. Dark exposure decreases calcium spike activity that leads to a decrease in the number of dopaminergic neurons. 
the resulting calcium transients mediate the activation of a transcription factor that regulates a downstream selector gene involved in the determination of neurotransmitter expression. These results illustrate the importance of activity-dependent transcriptional regulation in controlling neuronal differentiation.

Activity-dependent transcriptional regulation of neurotransmitter expression is not restricted to spinal neurons. Additional studies show that activity-dependent regulation of the transcription factor Lmxlb controls the extent of serotonergic specification in neurons present in the raphe nucleus of the Xenopus hindbrain (Demarque and Spitzer 2010). Increased calcium spiking results in a decrease in the number of neurons expressing Lmx1b and a corresponding decrease in the number of serotonergic neurons. In contrast, a decrease in calcium spiking activity leads to an increase in Lmx1b expression and an increase in the number of serotonergic neurons in the hindbrain. Importantly, this study shows that the activity-dependent induction of additional serotonergic neurons leads to a change in behavioral output-specifically in the swimming behavior of the Xenopus larvae. Serotonin has a key role in limiting the duration of swimming episodes (Sillar et al. 1998), suggesting that an increase in the number of serotonergic cells will lead to a decrease in the duration in swimming episodes. Consistent with this hypothesis, the presence of additional serotonergic neurons generated either by experimental reduction of calcium spiking or overexpression of Lmxlb is sufficient to reduce both the duration of fictive swimming firing patterns recorded from ventral nerve roots and the duration of swimming episodes performed by free swimming larvae (Demarque and Spitzer 2010). In contrast, either an increase in activity or a reduction in Lmxlb expression is sufficient to extend the duration of both fictive and free swimming episodes. These findings show that experimentally induced alterations in calcium spiking can modulate behavior by redirecting the differentiated neurotransmitter fate of neuronal subtypes.

The experimental manipulation of calcium spiking in developing Xenopus embryos raises the important question of whether the effects of activity on neurotransmitter phenotype are restricted to early embryonic spontaneous activity or whether these changes can also be mediated by experience-driven changes in activity occurring after synapse formation. Studies of the circuitry underlying the camouflage behavior of Xenopus larvae tested whether experience-mediated changes in calcium spiking can influence neurotransmitter expression. Exposure of larvae either to light or to a white background activates dopaminergic neurons in the ventral suprachiasmatic nucleus (VSC), which regulate the downstream pathways responsible for changes in skin pigmentation. Exposing larvae to light for a period of several hours leads to an increase in the number of dopaminergic neurons, which is blocked by pharmacological inhibition of calcium spiking in the VSC (Fig. 2C) (Dulcis and Spitzer 2008). This increase in the dopaminergic population occurs through the acquisition of dopamine expression by existing neurons and does not involve the generation of new neurons. These findings indicate that experience-driven calcium spiking is sufficient to alter the number of dopaminergic neurons, demonstrating that experience-mediated activity can also influence neurotransmitter phenotype. Importantly, these experience-generated dopaminergic neurons are functionally sufficient to mediate camouflage behavior after the selective elimination of the original dopaminergic population.

In addition to experience-mediated activity, the binding of extracellular ligands can also modulate intracellular calcium levels. Furthermore, extracellular retrograde signals from axonal targets have been shown to influence neurotransmitter specification (Schotzinger and Landis 1988, 1990). Is it possible that these signals mediate neurotransmitter specification via regulation of calcium signaling? In developing rodents, sympathetic neurons innervating the sweat glands and the periosteum are initially adrenergic. Following innervation, these neurons down-regulate noradrenaline and instead adopt a cholinergic phenotype. Soluble factors derived from the innervated target tissues contribute to this switch in neuronal identity 
(Habecker et al. 1997; Asmus et al. 2001). Although the exact identity of these soluble factors remains elusive, these signaling molecules appear to belong to the IL-6 family of neuropoietic cytokines (Rao and Landis 1990). The selective in vivo deletion of the neuropoietic cytokine receptor subunit gp130 in noradrenergic neurons results in a decrease in the number of cholinergic neurons innervating the sweat glands, although the total number of innervating neurons is unchanged (Stanke et al. 2006). Additional studies of hippocampal neurons show that elevated levels of IL- 6 both in vivo and in vitro modulate the expression of L-type calcium channels and alter the dynamics of calcium signaling and network activity (Nelson et al. 2004; Vereyken et al. 2007). These results indicate that signaling through the IL-6 receptor gp130 may regulate neurotransmitter phenotype by modulating calcium signaling.

\section{THE ROLE OF ACTIVITY AND CALCIUM SIGNALING IN DENDRITIC ARBORIZATION}

Establishment and plasticity of dendritic morphology represent important aspects of neuronal differentiation. The relative growth and branching of dendritic trees influences the number of synaptic connections that a given neuron can form. Subgroups of neurons are characterized by distinct dendritic morphologies, which include variations in both the length and number of dendritic branches (Parrish et al. 2007; Spruston 2008). A role for activitydependent regulation of dendritic arborization has been described for numerous neuronal subtypes (Wong and Ghosh 2002), and activitymediated calcium signaling has been implicated in regulating the plasticity of dendritic branching and growth. Increases in intracellular calcium levels, resulting from the activation of NMDA receptors, promote an increase in dendritic branching in both rodent and amphibian neurons (Rajan and Cline 1998; Chevaleyre et al. 2002).

In cultures of developing Xenopus tectal neurons, application of APV selectively disrupts NMDA-mediated transmission and decreases the rate of dendritic growth, limiting both the extension of existing branches and the addition of new branches (Rajan and Cline 1998). In more mature cultured tectal neurons, the activation of both NMDA and AMPA receptors is necessary for dendritic growth and the maintenance of existing branches. Furthermore, the stability of dendritic arbors in Xenopus tectal neurons in vivo is regulated by the activity of the calcium-activated kinase, calcium-calmodulindependent kinase II, CaMKII (Wu and Cline 1998). As tectal neurons mature and migrate away from their site of generation in the ventricular layer of the tectum, the rate of dendritic growth declines, coincident with an increase in the expression of CaMKII. Overexpression of constitutively active CaMKII in developing neurons leads to a decrease in the rate of dendritic growth. Additionally, more mature tectal neurons show enhanced dendritic growth in the presence of the CaMKII inhibitor, KN-93. CaMKII regulates the rate of dendritic growth by inhibiting the addition and turnover of new dendritic branches. These results prompt further inquiry into the mechanisms regulating CaMKII expression and activity, as well as the CaMKII substrates and downstream pathways controlling dendritic growth and plasticity.

CaMKII is expressed in a graded fashion in the Xenopus tectum, with high expression restricted to the rostral-lateral region and little to no expression in the caudal-medial region where tectal neurons are initially formed. The CaMKII expression gradient may be influenced by inhibitory factors expressed in the ventricular layers of the tectum that facilitate increased dendritic growth by restricting CaMKII expression in newly generated neurons. However, both monocular deprivation and the induction of neuronal hyperexcitability in rodents result in activity-dependent regulation of CaMKII expression (Hanson and Schulman 1992; Hudmon and Schulman 2002). The maturation and migration of Xenopus tectal neurons is accompanied by an increase in the density of AMPA receptors at developing synapses and a corresponding decline in the number of silent synapses containing predominantly NDMA receptors (Wu and Cline 1998). This developmental shift in neuronal signaling capacity 
enables alterations in the extent of calcium influx, which could directly influence the activity-dependent regulation of CaMKII expression. It seems plausible that the establishment of the tectal CaMKII expression gradient results from a combinatorial effect of activity-dependent and extrinsic molecular mechanisms, which can both exert a degree of transcriptional control.

Dendritic branching is also regulated by both depolarization and the application of the peptidergic signaling molecules oxytocin and vasopressin in rat supraoptic nucleus (SON) slice cultures (Chevaleyre et al. 2002). Increased dendritic branching in SON neurons can be induced by the application of either NMDA or oxytocin and vasopressin, as well as by depolarization with high levels of extracellular potassium. Additionally, the injection of newborn rat pups with antagonists to either NMDA receptors or oxytocin/vasopressin receptors leads to a decrease in dendritic branching in the SON. In slice cultures, the increase in dendritic branching mediated by NMDA, oxytocin/vasopressin, and depolarization, requires both the activity of voltage-gated calcium channels and the release of calcium from intracellular stores. However, depolarization in the presence of either NMDA or oxytocin/vasopressin antagonists is not sufficient to increase dendritic branching. This result indicates that the regulation of dendritic branching requires multiple intracellular events downstream from NMDA and peptide-mediated signaling. These findings underscore the likelihood of a combinatorial role for both activity-dependent and activityindependent mechanisms in the calciummediated regulation of dendritic growth and plasticity.

\section{CALCIUM-MEDIATED REGULATION OF AXON OUTGROWTH AND PATHFINDING}

Neurotransmitter expression and dendritic morphology represent distinguishing features of different neuronal subtypes. The location and identity of a neuron's downstream synaptic partners provide an additional basis for neuronal characterization. Calcium signaling contributes to the regulation of both the rate of axon outgrowth and the directional navigation of the growth cone en route to the axon's target.

Live imaging studies performed in the developing Xenopus spinal cord indicate that the rate of axon outgrowth is regulated by the frequency of spontaneous calcium transients in the growth cone (Gomez and Spitzer 1999). The neuronal subtypes evaluated, including sensory Rohon-Beard neurons, ventral motor neurons, and two different classes of interneurons, show distinct frequencies of calcium transients, which are correlated with different rates of axon outgrowth. Neurons showing a high frequency of calcium transients experience a slow rate of axon outgrowth, whereas neurons with low frequency calcium activity show rapid rates of extension. Blocking or increasing the frequency of these calcium waves shows that they are both necessary and sufficient to regulate axon outgrowth. The frequency of the calcium transients within a given neuronal subtype is not fixed, and instead is subject to change depending on the location of the growth cone. This observation is consistent with studies demonstrating that gradients of extracellular guidance cues influence the calcium activity within neuronal growth cones (Hong et al. 2000; Henley et al. 2004). Additionally, the Xenopus live imaging findings are supported by studies of cultured Xenopus spinal neurons, which show that an optimal frequency of calcium transients regulates neurite extension $(\mathrm{Gu}$ and Spitzer 1995).

Recent work reveals that calcium-mediated regulation of axon outgrowth can also be controlled by kainate receptor-activated signaling. In cultured hippocampal neurons, electrical activity acts downstream from kainate receptor activation to decrease the rate of growth cone extension and axon outgrowth (Ibarretxe et al. 2007). The removal of extracellular calcium and the depletion of neuronal intracellular calcium stores prevent the inhibition of axon outgrowth that occurs on kainate application. These results indicate that an increase in intracellular calcium is necessary for kainate receptor-mediated inhibition of axon outgrowth. 
Although kainate application and direct electrical stimulation inhibit growth cone motility in a significant percentage of cultured neurons, the growth rate of some cells remains unimpaired. This finding indicates that divergent neuronal subtypes within hippocampal cultures may respond to extracellular and activity-dependent cues in a heterogeneous fashion. Differential responses to activity-dependent signaling could allow neuronal subtypes to extend their axons to targets in disparate locations.

The selection of a neuron's target-whether it is a neuron or another cell type-is determined to a large extent by the direction of axon outgrowth, which is governed by the response of the axonal growth cone to extracellular axon guidance cues. Guidance molecules include both attractive and repulsive cues that interact with receptors on the growth cone to stimulate turning, which ultimately dictates the direction of growth. The selection of an axonal target is therefore heavily influenced by the specific repertoire of guidance receptors expressed by developing axons. How does the interaction between guidance cues and their corresponding receptors mediate directional changes in the trajectory of the growth cone? Extracellular gradients of both attractive and repulsive guidance cues have been shown to affect growth cone calcium and cyclic nucleotide levels differentially depending on whether the guidance cue is attractive or repulsive (Hong et al. 2000; Henley et al. 2004). Calcium transients in growth cone filopodia regulate turning onto preferred biological substrates (Gomez et al. 2001). Some of the downstream signaling mechanisms responsible for growth cone turning have recently been reviewed (Gomez and Zheng 2006; Zheng and Poo 2007; O'Donnell et al. 2009; Kolodkin and Tessier-Lavigne 2010). Neurotransmitters may also act as guidance molecules. Early studies show that growth cones can turn toward gradients of acetylcholine or glutamate in a calcium-dependent manner in vitro (Zheng et al. 1994, 1996). In vivo, endocannabinoids regulate connectivity in the rodent cortex (Berghuis et al. 2007) and serotonin modulation of responsiveness to Netrin-1 may account for its disruption of sensory maps of thalamocortical axons (Bonnin et al. 2007). However, the role of calcium signaling in these cases is unclear.

\section{THE RELATIONSHIP BETWEEN ACTIVITY AND ION CHANNEL EXPRESSION}

The studies described above indicate that activity-dependent calcium signaling plays a significant role in regulating multiple aspects of neuronal differentiation. Many of these studies suggest that the influx of calcium into the neuron plays a critical role in inducing the release of intracellular calcium stores, allowing the progression of subsequent downstream events that are required for differentiation. A common theme in the work described here is the importance of both the spatial and temporal pattern of calcium transients in providing specificity for calcium-mediated regulation of distinct elements of neuronal development. The expression patterns and subcellular locations of calcium-permeable channels are instrumental in determining the spatiotemporal dynamics of calcium influx. Calcium enters the cell primarily through voltage-sensitive calcium channels, ionotropic glutamate receptors, and transient receptor potential (TRP) channels. Understanding the mechanisms that regulate the expression and localization of these channels can provide additional insight into the process of neuronal differentiation and the generation of specific neuronal subtypes.

Many of the studies described in this review suggest that neuronal phenotype is less hardwired than previously thought and that activity-dependent events can modulate neuronal phenotype throughout an extended period of development. Developmental changes in the expression profile of calcium-permeable channels provide a potential mechanism by which calcium signaling could play different roles at different times in the development of a single cell or cell type. For example, developmental studies of cultured mouse hippocampal neurons show that $\mathrm{Ca}_{\mathrm{v}} 1.2$, the most abundant neuronal L-type calcium channel, is initially expressed throughout the entire neuron (Obermair et al. 2004). As the neuron develops, the 
expression of $\mathrm{Ca}_{\mathrm{v}} 1.2$ is down-regulated in the axon, particularly in the more distal parts. In the mature neuron, the expression of $\mathrm{Ca}_{\mathrm{v}} 1.2$ is restricted to the somatic and dendritic compartments of the cell. These observations are intriguing because they present a potential scenario in which the early expression of $\mathrm{Ca}_{\mathrm{v}} 1.2$ in the axon, particularly in the growth cone, could play a role in the regulation of calciummediated axonal guidance and outgrowth, in combination with TRP channels (Wang and Poo 2005). Temporal regulation of the expression of calcium-permeable channels could provide a useful strategy for selectively altering the nature of calcium signaling for different developmental events. Further studies are required to determine whether developmental changes in calcium channel expression patterns provide specificity for calcium-mediated effects on neuronal differentiation. It is also necessary to elucidate whether temporal changes in the expression of calcium channels occur in multiple neuronal subtypes, in addition to mouse hippocampal neurons.

Observation of transient expression of $\mathrm{Ca}_{\mathrm{v}} 1.2$ in the axons of hippocampal neurons prompts further inquiry into the mechanisms responsible for the compartment specific targeting and subsequent compartmental restriction of $\mathrm{Ca}_{\mathrm{v}}$ 1.2. The targeting of $\mathrm{Ca}_{\mathrm{v}} 1.2$ in cultured rat hippocampal neurons is regulated by the interaction of the $\mathrm{Ca}_{\mathrm{v}} \alpha 1 \mathrm{c}$ subunit with the calcium-binding protein, calmodulin (Wang et al. 2007). Intriguingly, the calmodulin-dependent trafficking of $\mathrm{Ca}_{\mathrm{v}} 1.2$ to distal dendrites is enhanced following up-regulation of intracellular calcium. Mechanistically, this provides a means by which calcium signaling in the neuron can regulate future calcium signaling events. For example, influx of calcium leading to a greater level of $\mathrm{Ca}_{\mathrm{v}} 1.2$ insertion in the membrane and trafficking to more distal dendrites, could act as a positive feedback mechanism to promote additional subtypespecific calcium-mediated events influencing dendritic development. In this way, calcium signaling could act as a positive feedback mechanism to regulate differentiation in a subset of developing cells, aiding the specification of different neuronal subtypes. Additionally, calcium-mediated changes in calcium channel trafficking could underlie activity-dependent changes in neurotransmitter phenotype. For instance, up-regulation of calcium signaling promoted by the introduction of voltage-gated sodium channel constructs could lead to an increase in the membrane targeting of $\mathrm{Ca}_{\mathrm{v}} 1.2$ channels. Increased calcium influx as a result of additional $\mathrm{Ca}_{\mathrm{v}} 1.2$ channels could evoke changes in excitation-transcriptional signaling that may be responsible for the phosphorylation of transcription factors like cJun and the activity-dependent expression of genes such as Tlx3. Future studies will delineate further roles of calcium signaling in neuronal development and shed light on the myriad ways in which the influx of calcium at the membrane can mediate changes in the expression of genes, the structure of the cytoskeleton, and the subcellular targeting of proteins such as ionpermeable channels.

\section{FREQUENCY CODING BY CALCIUM TRANSIENTS}

The distinct developmental roles of the calcium transients described above indicate that the temporal dynamics of calcium fluctuations are reliably decoded and translated into specific effects on neuronal differentiation. In the mature nervous system, neuronal action potentials, resulting from both evoked and spontaneous activity, are all-or-none events that are characterized by a stereotyped change in membrane polarization and ion flux. Because the magnitude of depolarization does not provide information about the nature of the stimulus, the nervous system often relies on frequency coding to translate the temporal patterns of spiking activity into useful information. Similarly, in the developing nervous system, the rate of neurite extension is regulated by the frequency of spontaneous calcium transients (Gu and Spitzer 1995). In addition, the frequency of calcium transients regulates the expression levels of GAD67 (an enzyme that synthesizes GABA), and the number of GABAergic neurons. 


\section{S.S. Rosenberg and N.C. Spitzer}

How is information encoded in the temporal pattern of action potential firing and calcium transients? Temporal variability is present in the duration of bursts of single spikes and in the length of time between bursts - the interburst interval. How do neurons decode these temporal features of spiking into effects on neuronal development and plasticity? Future studies deciphering neuronal decoding strategies will provide insight into the regulation of neuronal development and specification, as well the formation and plasticity of neuronal circuits.

\section{CONCLUDING THOUGHTS}

Elucidating the role of calcium signaling in neuronal development provides a better understanding of the formation and function of healthy neuronal circuits, and may also provide key insight into the pathogenesis of neurodegenerative and neurodevelopmental disorders. Parkinson's disease, Huntington's disease, and amyotrophic lateral sclerosis are characterized by the death or dysfunction of specific neuronal subtypes. Similarly, subtype-specific deficits have also been hypothesized to play a role in neurodevelopmental disorders such as autism and schizophrenia. Further exploration of the calcium-mediated mechanisms responsible for neuronal subtype specification may identify new treatments for neurological disorders.

\section{REFERENCES}

Asmus SE, Tian H, Landis SC. 2001. Induction of cholinergic function in cultured sympathetic neurons by periosteal cells: Cellular mechanisms. Dev Biol 235: $1-11$.

Berghuis P, Rajnicek AM, Morozov YM, Ross RA, Mulder J, Urban GM, Monory K, Marsicano G, Matteoli M, Canty A, et al. 2007. Hardwiring the brain: Endocannabinoids shape neuronal connectivity. Science 316: 1212-1216.

Blankenship AG, Feller MB. 2010. Mechanisms underlying spontaneous patterned activity in developing neural circuits. Nat Rev Neurosci 11: 18-29.

Bonnin A, Torii M, Wang L, Rakic P, Levitt P. 2007. Serotonin modulates the response of embryonic thalamocortical axons to netrin-1. Nat Neurosci 10: 588-597.

Borodinsky LN, Spitzer NC. 2007. Activity-dependent neurotransmitter-receptor matching at the neuromuscular junction. Proc Natl Acad Sci 104: 335-340.
Borodinsky LN, Root CM, Cronin JA, Sann SB, Gu X, Spitzer NC. 2004. Activity-dependent homeostatic specification of transmitter expression in embryonic neurons. Nature 429: 523-530.

Catsicas M, Bonness V, Becker D, Mobbs P. 1998. Spontaneous $\mathrm{Ca}^{2+}$ transients and their transmission in the developing chick retina. Curr Biol 8: 283-286.

Chang LW, Spitzer NC. 2009. Spontaneous calcium spike activity in embryonic spinal neurons is regulated by developmental expression of the $\mathrm{Na}^{+}, \mathrm{K}^{+}$-ATPase $\beta 3$ subunit. J Neurosci 29: 7877-7885.

Cheng L, Arata A, Mizuguchi R, Qian Y, Karunaratne A, Gray PA, Arata S, Shirasawa S, Bouchard M, Luo P, et al. 2004. $T l x 3$ and $T l x 1$ are post-mitotic selector genes determining glutamatergic over GABAergic cell fates. Nat Neurosci 7: 510-517.

Cheng L, Samad OA, Xu Y, Mizuguchi R, Luo P, Shirasawa S, Goulding M, Ma Q. 2005. Lbx1 and Tlx3 are opposing switches in determining GABAergic versus glutamatergic transmitter phenotypes. Nat Neurosci 8: 1510-1515.

Chevaleyre V, Moos FC, Desarmenien MG. 2002. Interplay between presynaptic and postsynaptic activities is required for dendritic plasticity and synaptogenesis in the supraoptic nucleus. J Neurosci 22: 265-273.

Demarque M, Spitzer NC. 2010. Activity-dependent expression of Lmxlb regulates specification of serotonergic neurons modulating swimming behavior. Neuron 67: 321-334.

Dulcis D, Spitzer NC. 2008. Illumination controls differentiation of dopamine neurons regulating behaviour. Nature 456: 195-201.

Gomez TM, Spitzer NC. 1999. In vivo regulation of axon extension and pathfinding by growth-cone calcium transients. Nature 397: 350-355.

Gomez TM, Zheng JQ. 2006. The molecular basis for calcium-dependent axon pathfinding. Nat Rev Neurosci 7: 115-125.

Gomez TM, Snow DM, Letourneau PC. 1995. Characterization of spontaneous calcium transients in nerve growth cones and their effect on growth cone migration. Neuron 14: $1233-1246$.

Gomez TM, Robles E, Poo M, Spitzer NC. 2001. Filopodial calcium transients promote substrate-dependent growth cone turning. Science 291: 1983-1987.

Gu X, Spitzer NC. 1995. Distinct aspects of neuronal differentiation encoded by frequency of spontaneous $\mathrm{Ca}^{2+}$ transients. Nature 375: 784-787.

Gu X, Spitzer NC. 1997. Breaking the code: Regulation of neuronal differentiation by spontaneous calcium transients. Dev Neurosci 19: 33-41.

Gu X, Olson EC, Spitzer NC. 1994. Spontaneous neuronal calcium spikes and waves during early differentiation. J Neurosci 14: 6325-6335.

Habecker BA, Symes AJ, Stahl N, Francis NJ, Economides A, Fink JS, Yancopoulos GD, Landis SC. 1997. A sweat gland-derived differentiation activity acts through known cytokine signaling pathways. J Biol Chem 272: 30421-30428.

Hanson PI, Schulman H. 1992. Neuronal $\mathrm{Ca}^{2+} /$ calmodulin-dependent protein kinases. Annu Rev Biochem 61: 559-601. 
Henley JR, Huang KH, Wang D, Poo MM. 2004. Calcium mediates bidirectional growth cone turning induced by myelin-associated glycoprotein. Neuron 44: 909-916.

Holliday J, Adams RJ, Sejnowski TJ, Spitzer NC. 1991. Calcium-induced release of calcium regulates differentiation of cultured spinal neurons. Neuron 7: 787-796.

Hong K, Nishiyama M, Henley J, Tessier-Lavigne M, Poo M. 2000. Calcium signalling in the guidance of nerve growth by netrin-1. Nature 403: 93-98.

Hudmon A, Schulman H. 2002. Neuronal $\mathrm{Ca}^{2+} /$ calmodulin-dependent protein kinase II: The role of structure and autoregulation in cellular function. Annu Rev Biochem 71: 473-510.

Ibarretxe G, Perrais D, Jaskolski F, Vimeney A, Mulle C. 2007. Fast regulation of axonal growth cone motility by electrical activity. J Neurosci 27: 7684-7695.

Kolodkin AL, Tessier-Lavigne M. 2010. Mechanisms and molecules of neuronal wiring: A primer. Cold Spring Harb Perspect Biol doi: 10.1101/cshperspect.a001727.

Marek KW, Kurtz LM, Spitzer NC. 2010. cJun integrates calcium activity and tlx3 expression to regulate neurotransmitter specification. Nat Neurosci 13: 944-950.

Nelson TE, Netzeband JG, Gruol DL. 2004. Chronic interleukin-6 exposure alters metabotropic glutamate receptor-activated calcium signalling in cerebellar Purkinje neurons. Eur J Neurosci 20: 2387-2400.

Obermair GJ, Szabo Z, Bourinet E, Flucher BE. 2004. Differential targeting of the L-type $\mathrm{Ca}^{2+}$ channel $\alpha 1 \mathrm{C}\left(\mathrm{Ca}_{\mathrm{V}} 1.2\right)$ to synaptic and extrasynaptic compartments in hippocampal neurons. Eur J Neurosci 19: 2109-2122.

O’Donnell M, Chance RK, Bashaw GJ. 2009. Axon growth and guidance: Receptor regulation and signal transduction. Annu Rev Neurosci 32: 383-412.

O’Donovan MJ. 1999. The origin of spontaneous activity in developing networks of the vertebrate nervous system. Curr Opin Neurobiol 9: 94-104.

Parrish JZ, Emoto K, Kim MD, Jan YN. 2007. Mechanisms that regulate establishment, maintenance, and remodeling of dendritic fields. Annu Rev Neurosci 30: 399-423.

Platel JC, Dave KA, Bordey A. 2008. Control of neuroblast production and migration by converging GABA and glutamate signals in the postnatal forebrain. J Physiol 586: 3739-3743.

Rajan I, Cline HT. 1998. Glutamate receptor activity is required for normal development of tectal cell dendrites in vivo. J Neurosci 18: 7836-7846.

Rao MS, Landis SC. 1990. Characterization of a targetderived neuronal cholinergic differentiation factor. $\mathrm{Neu}$ ron 5: 899-910.

Root CM, Velazquez-Ulloa NA, Monsalve GC, Minakova E, Spitzer NC. 2008. Embryonically expressed GABA and glutamate drive electrical activity regulating neurotransmitter specification. J Neurosci 28: 4777-4784.

Schotzinger RJ, Landis SC. 1988. Cholinergic phenotype developed by noradrenergic sympathetic neurons after
Calcium Signaling in Neuronal Development

innervation of a novel cholinergic target in vivo. Nature 335: 637-639.

Schotzinger RJ, Landis SC. 1990. Acquisition of cholinergic and peptidergic properties by sympathetic innervation of rat sweat glands requires interaction with normal target. Neuron 5: $91-100$.

Sillar KT, Reith CA, McDearmid JR. 1998. Development and aminergic neuromodulation of a spinal locomotor network controlling swimming in Xenopus larvae. Ann NY Acad Sci 860: 318-332.

Spitzer NC. 2006. Electrical activity in early neuronal development. Nature 444: 707-712.

Spitzer NC, Gu X, Olson E. 1994. Action potentials, calcium transients and the control of differentiation of excitable cells. Curr Opin Neurobiol 4: 70-77.

Spruston N. 2008. Pyramidal neurons: Dendritic structure and synaptic integration. Nat Rev Neurosci 9: 206-221.

Stanke M, Duong CV, Pape M, Geissen M, Burbach G, Deller T, Gascan H, Otto C, Parlato R, Schutz G, et al. 2006. Target-dependent specification of the neurotransmitter phenotype: Cholinergic differentiation of sympathetic neurons is mediated in vivo by gp 130 signaling. Development 133: $141-150$.

Vereyken EJ, Bajova H, Chow S, de Graan PN, Gruol DL. 2007. Chronic interleukin-6 alters the level of synaptic proteins in hippocampus in culture and in vivo. Eur $J$ Neurosci 25: 3605-3616.

Wang GX, Poo MM. 2005. Requirement of TRPC channels in netrin-1-induced chemotropic turning of nerve growth cones. Nature 434: 898-904.

Wang HG, George MS, Kim J, Wang C, Pitt GS. 2007. $\mathrm{Ca}^{2+} /$ calmodulin regulates trafficking of $\mathrm{Ca}_{\mathrm{V}} 1.2 \mathrm{Ca}^{2+}$ channels in cultured hippocampal neurons. J Neurosci 27: 9086-9093.

Watt SD, Gu X, Smith RD, Spitzer NC. 2000. Specific frequencies of spontaneous $\mathrm{Ca}^{2+}$ transients upregulate GAD 67 transcripts in embryonic spinal neurons. Mol Cell Neurosci 16: 376-387.

Wong RO, Ghosh A. 2002. Activity-dependent regulation of dendritic growth and patterning. Nat Rev Neurosci 3: 803-812.

Wu GY, Cline HT. 1998. Stabilization of dendritic arbor structure in vivo by CaMKII. Science 279: 222-226.

Yuste R, Peinado A, Katz LC. 1992. Neuronal domains in developing neocortex. Science 257: 665-669.

Zheng JQ, Poo MM. 2007. Calcium signaling in neuronal motility. Annu Rev Cell Dev Biol 23: 375-404.

Zheng JQ, Felder M, Connor JA, Poo MM. 1994. Turning of nerve growth cones induced by neurotransmitters. Nature 368: 140-144.

Zheng JQ, Wan JJ, Poo MM. 1996. Essential role of filopodia in chemotropic turning of nerve growth cone induced by a glutamate gradient. J Neurosci 16: 1140-1149. 


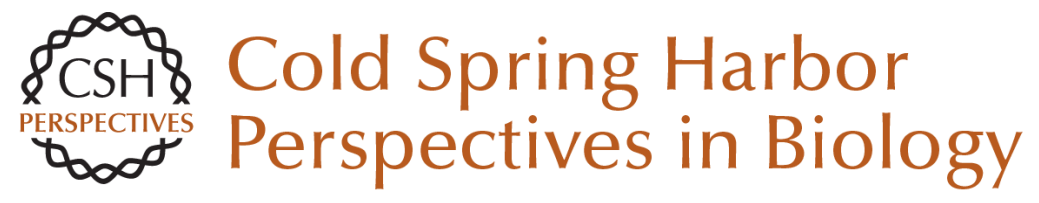

\section{Calcium Signaling in Neuronal Development}

Sheila S. Rosenberg and Nicholas C. Spitzer

Cold Spring Harb Perspect Biol 2011; doi: 10.1101/cshperspect.a004259 originally published online July 5, 2011

\section{Subject Collection Calcium Signaling}

The Endoplasmic Reticulum-Plasma Membrane Junction: A Hub for Agonist Regulation of $\mathrm{Ca}^{2+}$ Entry

Hwei Ling Ong and Indu Suresh Ambudkar

Calcium-Handling Defects and Neurodegenerative Disease

Sean Schrank, Nikki Barrington and Grace E. Stutzmann

Lysosomal $\mathrm{Ca}^{2+}$ Homeostasis and Signaling in Health and Disease

Emyr Lloyd-Evans and Helen Waller-Evans

\section{$\mathrm{Ca}^{2+}$ Signaling in Exocrine Cells}

Malini Ahuja, Woo Young Chung, Wei-Yin Lin, et al.

Functional Consequences of Calcium-Dependent Synapse-to-Nucleus Communication: Focus on Transcription-Dependent Metabolic Plasticity Anna M. Hagenston, Hilmar Bading and Carlos Bas-Orth

Identifying New Substrates and Functions for an Old Enzyme: Calcineurin Jagoree Roy and Martha S. Cyert

Fundamentals of Cellular Calcium Signaling: A Primer

Martin D. Bootman and Geert Bultynck
Primary Active $\mathrm{Ca}^{2+}$ Transport Systems in Health and Disease Jialin Chen, Aljona Sitsel, Veronick Benoy, et al.

Signaling through $\mathrm{Ca}^{2+}$ Microdomains from Store-Operated CRAC Channels Pradeep Barak and Anant B. Parekh

Structural Insights into the Regulation of $\mathrm{Ca}^{2+}$ /Calmodulin-Dependent Protein Kinase II (CaMKII) Moitrayee Bhattacharyya, Deepti Karandur and John Kuriyan

Store-Operated Calcium Channels: From Function to Structure and Back Again Richard S. Lewis

Bcl-2-Protein Family as Modulators of $\mathrm{IP}_{3}$

Receptors and Other Organellar $\mathrm{Ca}^{2+}$ Channels Hristina Ivanova, Tim Vervliet, Giovanni Monaco, et al.

Calcium Signaling in Cardiomyocyte Function Guillaume Gilbert, Kateryna Demydenko, Eef Dries, et al.

Cytosolic $\mathrm{Ca}^{2+}$ Buffers Are Inherently $\mathrm{Ca}^{2+}$ Signal Modulators Beat Schwaller

For additional articles in this collection, see http://cshperspectives.cshlp.org/cgi/collection/

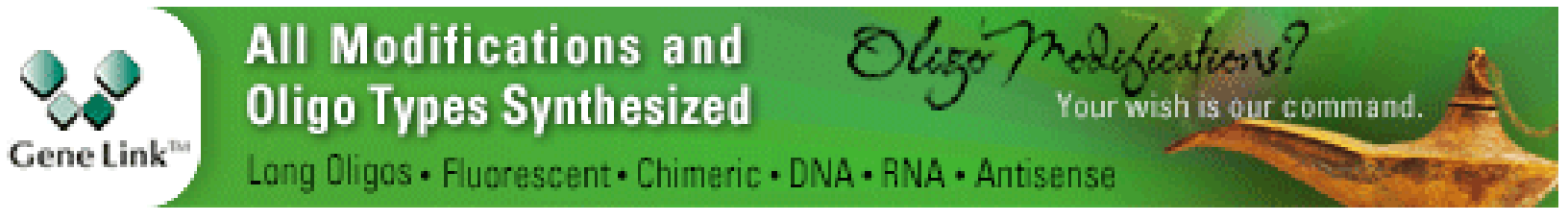


Role of Two-Pore Channels in Embryonic Development and Cellular Differentiation Sarah E. Webb, Jeffrey J. Kelu and Andrew L. Miller

\section{Organellar Calcium Handling in the Cellular \\ Reticular Network}

Wen-An Wang, Luis B. Agellon and Marek Michalak

For additional articles in this collection, see http://cshperspectives.cshlp.org/cgi/collection/

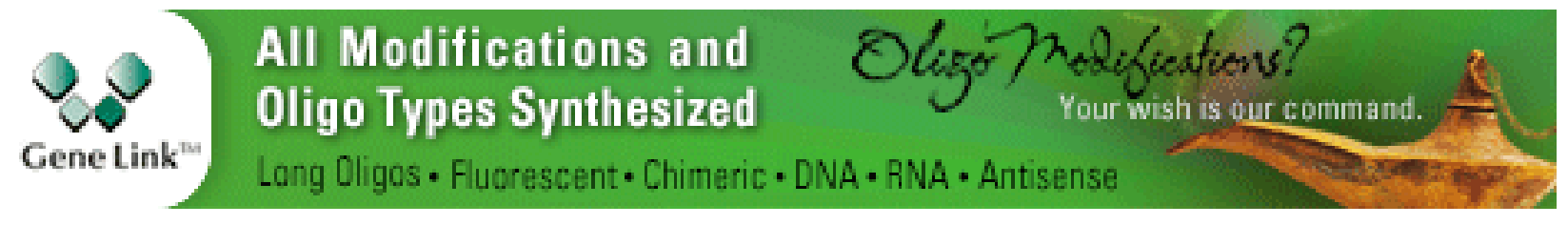

Copyright @ 2011 Cold Spring Harbor Laboratory Press; all rights reserved 